\title{
Experimental study of aerobic exercise on the weight loss effect of obese female college students.
}

\author{
Guo Siqiang* \\ College of Physical Education and Health, Zhaoqing University, Zhaoqing 526061, Guangdong, PR China
}

\begin{abstract}
Objective: To observe the effect of aerobic exercise on the weight loss effect of obese female students. Methods: 100 cases of female college students with weight over $58 \mathrm{~kg}$ were selected and evenly divided into two groups. The experimental group took aerobic exercise while the control group was given oral calcium pyruvate to lose weight. The physiological and biochemical indicators of both groups before and after experiment were observed.

Results: After the experiment, the weight, body fat content, WHR, BMI, and blood lipid level of both groups reduced significantly. The differences were statistically significant, with $P<0.05$. The effect of the experimental group was significantly better than the control group. The weight loss and physical and mental health condition of the experimental group were better than the control group. The difference was significant, with $\mathbf{P}<0.05$.

Conclusion: Aerobic exercise shows good effect on the weight loss of female college students, with promoting their physical and mental health. The significant effect of aerobic exercise makes it worth clinical promotion.
\end{abstract}

Keywords: Aerobic exercise, Obese female college students, Weight loss effect.

Accepted on April 25, 2017

\section{Introduction}

The constant development of economic level and corresponding changes of life style, we are facing gradually increasing weight, with younger-age trend. As a common metabolic disease in modern society, obesity is mostly caused by unreasonable lifestyle and eating habits. Obesity affects people's health greatly in figures and images as well as diseases including high blood pressure, diabetes, and various cardiovascular diseases [1]. As for female college students, the influence is more in job hunting and dating. Some of them failed to find a satisfied job or a good date because of obesity. As one of the most effective way against obesity, exercise, especially aerobatic exercise, can help reduce fat, lose weight, and change appearance [2]. 100 cases of female college students who are over $58 \mathrm{~kg}$ were selected and equally divided into two groups of different ways to lose weight. The effects of both groups were observed. Refer the following for details:

\section{Objects and Methods}

\section{Objects}

100 cases of female college students who were over $58 \mathrm{~kg}$ were selected as research objects and evenly divided into two groups. In the experimental group, the age range is 19 to 24 years old, with average age of $20.5 \pm 1.5$ years old; and the weight range is 58 to $68 \mathrm{~kg}$, with average weight of $61.5 \pm 1.5$ $\mathrm{kg}$. In the control group, the age range is 20 to 25 years old, with average age of $20.5 \pm 2.5$ years old; and the weight range is 58.6 to $66.4 \mathrm{~kg}$, with average weight of $60.5 \pm 2.5 \mathrm{~kg}$. After the comparison of age and weight between both groups, it was found that the data difference was not significant, with $\mathrm{P}>0.05$. Therefore, we can use these data for comparison.

\section{Methods}

\section{Literature}

The reference of a large number of research results and documentation related to aerobic exercise, exercise, and weight loss through the Internet laid a good foundation for this research.

\section{Experiment}

Weight, waist circumference, chest circumference, hip circumference, skinfold thickness, endurance test, strength test, and flexibility of all research objects were tested. Then the experimental group took aerobic exercise. Exercise intensity: moderate intensity, with heart rate being controlled in 120-150/ min. Exercise amount: 60 minutes each time, and 4 times per week. The exercise was divided into two: one in the morning for half an hour. Students could do rope skipping lasting 10 minutes for three times; another in the afternoon. Students could swim for half an hour continuously [3]. The training 
could be adjusted based on the conditions of different students. Generally, the training should be done four times a week for 12 weeks. Two or three items could be done at one time [4]. The control group was given oral Calcium pyruvate (produced by Shanghai Taiyangshen Hi-Tech Co., Ltd) of $50 \mathrm{mg} / \mathrm{kg}$ twice a day for 12 weeks. During this period, all students in the control groups should not take any other drugs, and the content of Calcium pyruvate should be more than $99 \%$ [5].

Various examinations including weight, BMI, body fat, and waist-to-hip ratio are given to all objects with body content analyzer. Lance clamp crease instrument is used to measure the TSF, SBS, and abdominal skinfold thickness. Blood examine index: Before exercise, $5 \mathrm{ml}$ venous blood of all objects is obtained in the resting state. The automatic biochemical analyzer was used to test the TG, TC, HDL-C, and LDL-C of all objects [6].

The physiological and biochemical indicators of both groups before and after experiment were observed.

\section{Mathematical statistics}

SPSS20.0 was used to calculate the indicators. Weight, BMI, body fat, waist-to-hip ratio, TSF, SBS, abdominal skinfold thickness, TG, TC, HDL-C, and LDL-C are measurement data. If there were significant differences between the data of both groups, $\mathrm{P}<0.05$.

\section{Results and Analysis}

\section{Comparison of weight, BMI, body fat, and WHR of both groups before and after experiment}

The weight, BMI, body fat, and WHR of both groups before and after experiment were compared. It was found that the weight, BMI, body fat, and WHR of both groups were reduced than before. The change of the experimental groups was greater. The weight, BMI, body fat, and WHR of the experimental group were significantly lower than the control group. The differences were statistically significant, with $\mathrm{P}<0.05$, as is shown in Table 1 .

\section{Comparison of TSF, SBS, and skinfold thickness of both groups after the experiment}

The TSF, SBS, and skinfold thickness of both groups after the experiment were compared. Compared with the control group, the skinfold thickness thickening of the experimental group was larger. The difference was statistically significant, with $\mathrm{P}<0.05$, as is shown in Table 2 .

\section{Comparison of TG, TC, HDL-C, and LDL-C changes of both groups after the experiment}

The TG, TC, HDL-C, and LDL-C of both groups after the experiment were compared. The TG values of both groups showed significant difference, with $\mathrm{P}<0.05$. While other indicators showed no significant differences, with $\mathrm{P}>0.05$, as is shown in Table 3.

\section{Analysis}

As is shown from the above indicators, aerobic exercise can help reduce the weight, BMI, body fat, and WHR of students, reduce TSF, SBS, abdominal skinfold thickness, and triglyceride, which were good for their weight loss.

Table 1. Comparison of weight, BMI, body fat, and WHR of female students in both groups $(\bar{x} \pm s)$.

\begin{tabular}{|c|c|c|c|c|}
\hline \multirow[t]{2}{*}{ Item } & \multicolumn{2}{|l|}{ Control group } & \multicolumn{2}{|l|}{ Experimental group } \\
\hline & Before the experiment & After the experiment & Before the experiment & After the experiment \\
\hline Weight (kg) & $66.8 \pm 7.2$ & $60.6 \pm 8.4$ & $70.6 \pm 6.2$ & $58.7 \pm 7.1$ \\
\hline BMI & $26.8 \pm 3.4$ & $24.1 \pm 2.9$ & $25.5 \pm 2.7$ & $21.6 \pm 3.1$ \\
\hline Body fat (\%) & $27.5 \pm 6.4$ & $22.8 \pm 5.9$ & $29.4 \pm 6.6$ & $21.7 \pm 5.9$ \\
\hline Waist-to-hip ratio & $0.86 \pm 0.08$ & $0.78 \pm 0.06$ & $0.92 \pm 0.07$ & $0.75 \pm 0.08$ \\
\hline
\end{tabular}

Note: Compared with the control group, the decline degree of the experimental group was more significant. The difference was statistically significant, with $\mathrm{P}<0.05$.

Table 2. Comparison of TSF, SBS, and skinfold thickness of both groups after the experiment $(\bar{x} \pm s)$.

\begin{tabular}{lllll}
\hline Group & $\mathrm{n}$ & TSF $(\mathrm{mm})$ & SBS $(\mathrm{mm})$ & Abdominal Skinfold Thickness $(\mathrm{mm})$ \\
\hline Experimental group & 50 & $3.69 \pm 1.34$ & $3.92 \pm 2.12$ & $5.32 \pm 1.48$ \\
\hline Control group & 50 & $4.98 \pm 1.07$ & $4.83 \pm 2.04$ & $7.28 \pm 1.39$ \\
\hline $\mathrm{t}$ & -- & 5.319 & 2.187 & 6.8259 \\
\hline
\end{tabular}




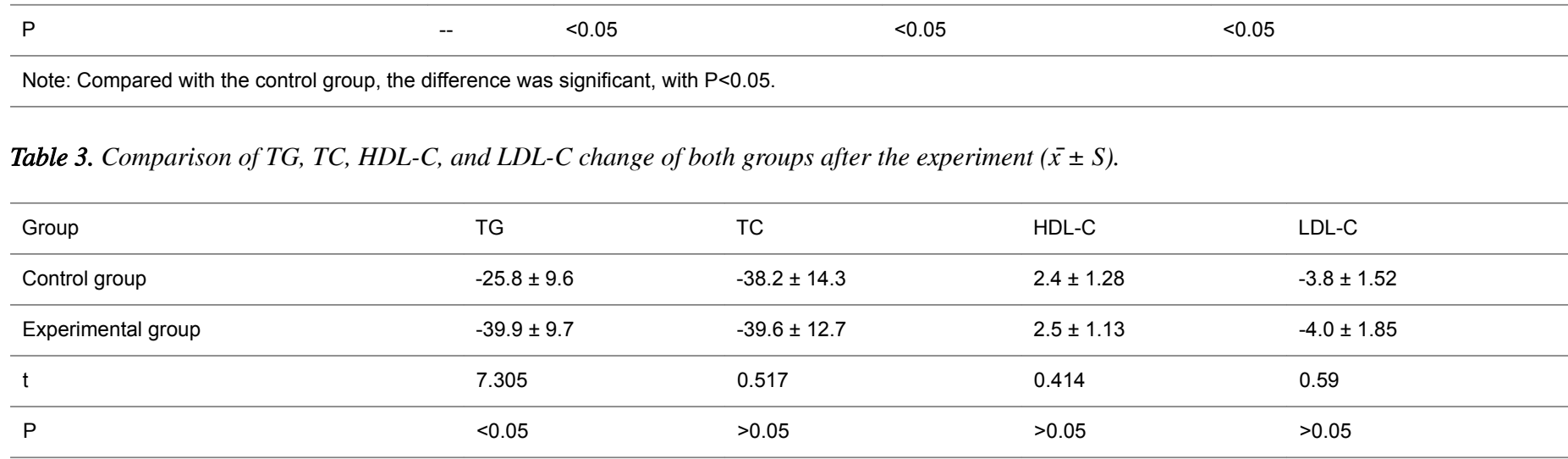

Note: The difference of TG between the experimental group and control group was significant, with $\mathrm{P}<0.05$.

\section{Conclusion}

Obesity refers to the state caused by overweight or excessive body fat. It is not just weight gain, but the excessive accumulation of body fat. Obesity is a pathologic and physiological phenomenon caused by excessive food intake or metabolic problems. Obesity is caused by complicated factors including genetic factors, environment, metabolism, endocrine function change, excessive fat, lifestyle and eating habits, drug-induced obesity, and intestinal problems [7]. Simple obesity accounts for $95 \%$ in all. "Middle age obesity" also belongs to simple obesity. Normally, the sugar, fat, and calorie in the daily diet are high. Once the intake of fat and sugar is greater than the body can consume, the excessive fat and sugar in the body will accumulate. At the same time, the excessive intake of alcohol will also cause excess energy and leads to obesity [8]. According to the reports in recent years, some girls would take cosmetic surgery or liposuction for beautiful figures. Some survey shows that there are foreign female college students who would get rid of their ribs for better figures. A large number of men also take liposuction to lose weight because obesity has endangered their health. The effect of obesity on human body is great, especially for female college students. Female college students are in their best days of life. However, obesity can damage their figure; affect their job hunting after graduation. Some of them even lost their jobs because of obesity. Therefore, it is good for fat metabolism of female college students to control their weight, build good eating and living habits, and keep exercising $[9,10]$.

Calcium pyruvate is a calcium salt as well as a dietary supplement. It can accelerate fat consumption, help reduce weight, enhance endurance, and promote effectiveness of athleticism. What's more, it can protect our heart and enhance the function of heart muscle [11]. According to the market research in China, however, many female college students would rather taking drugs such as L-carnitine and slimming tea, rather than taking exercise. These drugs often fail to lose weight, but bring some side effects. Aerobic exercise means exercises in the case of adequate oxygen. For people who want to lose weight, taking aerobic exercise is a long-term plan. Common aerobic exercises are: walking, jogging, climbing, rope skipping, swimming, riding bikes, fit aerobics, and yoga. Aerobic exercise can consume body fat, which in turn brings good weight loss effect. Therefore, most people take this way to lose weight. Aerobic exercise can increase blood flow and oxygen transmission capacity, and promote blood circulation and inner metabolism. It can also help enhance the function of heart and lung, increase bone density to prevent from osteoporosis, fight against aging, prevent the happening of diseases, and help improve attitude to keep good mood [12]. Therefore, aerobic exercise is suitable for female college students to lose weight. Aerobic exercise can help enhance their physical fitness and shape their shape, which is good for their physical and mental health.

Compared with drugs, the effect of aerobic exercises is relatively slow. But it has no side effects, with enriching students' after school life for better work and rest schedule. The effect is significant. On the other hand, taking drugs to lose weight has a great possibility of rebound, with strong stimulation of the gastrointestinal tract. Long-term use of drugs may lead to unhealthy physical and mental condition [13].

Above all, obesity has a large impact on female college students. Some of them will buy drugs due to unwillingness of exercise. However, the drugs for losing weight often has great side effect and easy to rebound later, with damage to physical and mental health. Aerobic exercise can help consume fat through exercise, which can help students relax, with little risk of rebound. Therefore, aerobic exercise is more suitable for obese female college students to lose weight.

\section{References}

1. Yingxue C, Haixia Y. Study on the effect of aerobic exercise on obese college students. Contemp Sports Sci 2015; 17-18.

2. Ting L. Effects of aerobic exercise and diet intervention on the physique and psychological health of obese middle school students. Contemp Sports Sci 2015; 5: 19-20.

3. Jimei S, Zelin Z. Efficacies of low-level laser and 1carnitine supplementation combined with aerobic exercise 
for weight loss in obese female undergraduate students. Laser J 2015; 36: 118-122.

4. Beibei L. Effects of aerobic exercise on obese university female students' body morphology, physiological functions, and blood lipid. J Shan Nor Univ 2015; 31: 131-134.

5. Tianxiao L, Jia L, Guanhua Z. Aerobic exercise with cassia tea for female college student's body shape and lipid metabolism. Shand Sports Sci Technol 2012; 34: 34-38.

6. Jianhua H. Effects on 8 weeks diet and exercise intervention on physiological parameters, blood lipid, and insulin of obese female college students. Zhej Sport Sci 2013; 35: 124-128.

7. Xia Y. Effects of exercise on the body morphology and heart rate of obese female college students. J Dalian Univ 2013; 34: 101-103.

8. Kailai H. Aerobic exercise combined with low resistance training study of obese female college students result reducing weight technique of practical fighting. Wushu Science 2013; 10: 122-124.

9. Baitong L, Yujiao L. Effects of aerobic exercise and resistance training on the body composition. body morphology, and physical indicators of obese female college students. Sports 2013; 64: 39-40.
10. Yugang Q, Jinhong H, Sijie T. Comparison of weight loss effects carried out by hiit and continuous aerobic exercise of female obese college students. Chin Sport Sci Technol 2013; 49: 30-33.

11. Huijun L, Haixia Y, Yingxue C. Study on the effect of weight reduction by exercise on obese college students' body morphology, physical quality, and physiological functions. J Inn Mongol Univ 2015; 6: 659-663.

12. Sheng M. Effect of aerobic exercise on the maximum oxygen uptake of obese college students. Tech Pract Fight 2015; 10: 12-13.

13. Beibei L, Anli H, Yang G. Effects of 4 weeks aerobic exercise on physique of obese adolescents. J Neij Norm Univ 2014; 12: 108-112.

\section{*Correspondence to:}

Guo Siqiang

College of Physical Education and Health

Zhaoqing University

PR China 